Mots. Les langages du politique

Ville et proximité. Le point de vue d'un géographeurbaniste

\title{
Remy Allain
}

\section{Q OpenEdition}

Journals

Édition électronique

URL : https://journals.openedition.org/mots/222

DOI : $10.4000 /$ mots.222

ISSN : 1960-6001

Éditeur

ENS Éditions

\section{Édition imprimée}

Date de publication : 1 mars 2005

Pagination : 129-136

ISBN : 2-84788-077-1

ISSN : 0243-6450

\section{Référence électronique}

Remy Allain, «Ville et proximité. Le point de vue d'un géographe-urbaniste », Mots. Les langages du politique [En ligne], 77 | 2005, mis en ligne le 31 janvier 2008, consulté le 22 avril 2022. URL : http:// journals.openedition.org/mots/222 ; DOI : https://doi.org/10.4000/mots.222 


\section{Remy Allain}

\section{Ville et proximité. Le point de vue d'un géographe-urbaniste}

Rien de plus banal et de plus simple en apparence que le mot «proximité». Or paradoxalement, alors que son importance relève de l'évidence dans les champs disciplinaires ayant l'espace ou des objets spatiaux au cœur de leurs préoccupations, il est peu présent dans le vocabulaire des géographes ou tout au moins dans leurs dictionnaires. Sans doute en raison de la banalité et de l'ambigüité du mot. Mais le duo conceptuel proximité-distance est bien sousjacent à la plupart de leurs modèles théoriques; il imprègne la réflexion normative sur l'aménagement de l'espace et notamment toute la pensée sur la ville. En tant que variable explicative ou causale, il intervient dans quantité de concepts servant à la justification théorique de la ville comme concentration spatiale, territoire censé regrouper des avantages tels qu'on ne peut que chercher à s'en rapprocher, fût-ce à un cout élevé. Mais de quelle proximité parle-ton, et pour qui ?

\section{La proximité, justification de la ville}

Si les hommes s'obstinent à se rassembler dans les villes, c'est qu'en dépit des inconvénients inhérents à la concentration, ils y trouvent des avantages. La ville est, en effet, une machine économique qui engendre une richesse supérieure à la somme de celles produites par l'ensemble de ses acteurs. L'idée est à la base du concept d'économies externes d'agglomération d'Alfred Marshall (1890) fondé sur la notion de proximité. Mais la rationalité de la relation économique entre proximité maximale et efficacité apparaissait déjà chez Smith et Condillac (1776). La ville permet aussi l'optimisation du marché par la rencontre des offres et des demandes en un même lieu. D'où l'image de commutateur central emprunté au langage des télécommunications (Claval, 1981). Dans un espace, le nombre de liaisons théoriques $L$ entre tous les lieux dispersés $n$ est $L=n(n-1) / 2$, soit vingt-et-une «routes» pour sept lieux. Une organisation polarisée autour d'un lieu central, autrement dit d'une ville, les réduit à six.

Université Rennes 2, RESO-CNRS, remy.allain@uhb.fr 
Le constat est pertinent à toutes les échelles. Les commerces se localisent en fonction du critère de la distance, définie comme le rayon des aires de chalandise et de l'accessibilité (centralité). Le modèle gravitaire de Willian J. Reilly (1931) emprunte à la loi de Newton l'idée que les interactions entre deux centres sont proportionnelles au produit de leur population et inversement proportionnelles au carré de la distance qui les sépare et qui peut être aussi la durée du trajet. Si Walter Christaller (1933) précise les lois de répartition des villes fondées sur le principe d'accessibilité aux biens et services (proximité), sa théorie comporte d'implicites propositions normatives pour un aménagement équilibré de l'espace qui ont inspiré beaucoup de pays.

Mais c'est au nom de l'efficacité que cet équilibre spatial hiérarchique est remis en cause par la métropolisation, c'est-à-dire la concentration de la population, des investissements et des activités, notamment directionnelles, dans les plus grandes villes où la proximité est maximale. La base théorique sert malheureusement d'excuse à l'abdication des scientifiques et des politiques devant cette tendance lourde à la «métropolisation du monde » accélérée par la globalisation.

La proximité est plus que jamais perçue comme nécessaire dans les activités de tertiaire supérieur ou décisionnel des métropoles. Ce qui ne signifie pas nécessairement proximité entre pouvoir économique et pouvoir politique, entre pouvoir réel et pouvoir symbolique. De ce point de vue, John Dos Passos, dans Manhattan Transfer, prête à l'un de ses personnages une des meilleures définitions du centre :

Pourquoi irais-je à Washington barboter dans ces eaux sales quand je suis sur la place même où on donne les ordres ? Ce qu'il y a de plus terrible quand on ne peut plus supporter New York, c'est qu'on ne sait pas où aller. C'est le sommet du monde. (A. Reynaud, 1981)

L'Astrée d'Honoré d'Urfé évoquait déjà le déplacement des lieux de pouvoir (Elias, 1969). Aujourd'hui, il ne s'agit plus de celui des nobles devant le Roi, mais des acteurs du jeu économique devant la Bourse et les institutions financières. La présence au centre, c'est donc d'abord la proximité avec les gens et les activités qui comptent.

\section{La proximité dans la ville : la centralité et ses contradictions}

La ville est aussi un espace foncier consommé dont le centre est doté des plus grands avantages, symbole d'une proximité entendue comme densité maximale d'interactions. La compétition pour le centre détermine alors la zonation concentrique des usages, des prix du sol (Alonso, 1964) et, par conséquent, des densités de bâti et des formes urbaines. Car la rentabilité de la consom- 
mation d'espace (ou le retour sur investissement) est directement liée aux deux variables que sont la distance et la situation. Le centre des affaires, cœur du downtown verticalisé, "la plus centrale des places centrales», est l'image de la proximité maximale. La réalité est un peu plus complexe, mais dans ces logiques, la fonction résidentielle est toujours perdante et le rôle social de la ville n'est plus prioritaire.

La ville est pourtant aussi le lieu de l'échange social. L'agora, lieu du débat entre citoyens, a matérialisé cette coïncidence entre politique et espace. Mais la nécessité du rassemblement physique, de la proximité spatiale, s'est progressivement affaiblie. Elle serait même devenue obsolète du fait du progrès des transports, de la télévision, de la télématique; l'espace public aurait quitté la place urbaine et même la ville pour le village-monde ou l'espace virtuel du web.

Au même titre que la vitalité des centres d'affaires (CBD), les phénomènes de polarisation dans la création culturelle et artistique prouvent pourtant le contraire. Le «face à face», la proximité physique demeurent essentiels. Le progrès des communications n'implique donc pas la «fin de la distance ». La centralité est bien plus qu'un simple marché d'idées ou d'informations obtenues par un clic sur une souris. Elle est un catalyseur d'innovations. «La ville ne crée rien et cependant elle crée tout, la situation urbaine, celle où les choses différentes adviennent les unes aux autres et n'existent pas séparément mais selon les différences, car précisément, elle les réunit» (Lefebvre, 1971).

La centralité n'existe pas sans une certaine identité, nulle part plus forte que dans le centre et dans ses espaces publics, rues et places. La forme urbaine participe donc à l'affirmation de la proximité : le centre doit sa reconnaissance à son rôle symbolique, fruit de l'histoire accumulée, et à la convergence des voies qui accentue celle des flux. La ville se définit par l'intensité ou la virtualité des interactions potentielles, des rencontres fortuites agréables, cette serendipity qui est la quintessence de l'urbanité, celle des piazzas italiennes ou des boulevards parisiens.

Tout le monde n'a cependant pas accès au centre, tout au moins pas à un même degré : les prix du foncier rejettent les habitants les plus modestes vers des périphéries de plus en plus éloignées. La vitesse des transports ne compense pas ce handicap, surtout dans les mégapoles où la ségrégation spatiale se manifeste sous des formes impitoyables. Le centre est en théorie le lieu le plus accessible pour le plus grand nombre. Certes, les déséconomies liées à l'engorgement ont conduit à l'éclatement de cette centralité. Mais les centralités périphériques (celles des Edge-Cities américaines, par exemple) n’existent que par référence à la ville-centre et elles sont toujours d'un niveau inférieur, notamment sur le plan de l'urbanité. 
Dès lors, deux questions se posent : celle du monopole et de la confiscation de la centralité (Lefebvre, 1968) qui émerge à partir d'un certain stade d'accumulation, et celle de l'obsolescence d'une certaine centralité du fait des progrès des transports et des communications qui déspatialisent l'urbain (Webber, 1964).

La réponse à la première interrogation passe par des politiques volontaires d'intervention sur l'espace «classique» : politique de mixité ou polycentrisme urbain permettent d'assurer une proximité minimale à l'ensemble d'un espace, conciliant efficacité, justice spatiale et urbanité.

La seconde question a conduit à conclure à l'émergence d'un espace «afocal», dépourvu de centre, qui caractériserait la nouvelle réalité de l'urbain et qui permettrait de dépasser les insolubles contradictions de la ville spatialisée. Cette vision est très influencée par la réalité nord-américaine (Los Angeles) et sa tradition de méfiance à l'égard de la ville. Elle néglige complètement la dimension morphologique de la ville en tant que «fait de culture». Ce n'est d'ailleurs pas un hasard si, depuis les années quatre-vingt, un courant contraire prône la défense de la ville compacte à l'européenne (Rapport Rogers, 1999, qui a influencé la loi SRU - solidarité et renouvellement urbain -, 2000, en France). Dans les deux cas, la question centrale devient alors celle de la mobilité.

\section{Mobilité et proximité. Les mots et les images}

La mobilité a contribué à relativiser la notion de proximité. Sa perception a été bouleversée par la rapidité des mutations des systèmes de transports. La vitesse croissante des déplacements a rétréci notre espace perçu (Ollivro, 2000). Notre «conscience du monde» en est transformée (Virilio, 1996). Des recherches ont montré de manière quantifiée à quel point la perception du proche et du lointain pouvait différer selon le vécu, le milieu social, la culture, la pratique quotidienne de l'espace. L'homme vit à l'intérieur de «coquilles» (Moles, 1977), enveloppes concentriques dont le rayon perçu diminue de manière logarithmique avec la distance. C'est dans les espaces proches que les choix sont les plus draconiens. Un habitant du centre d'une grande ville peut considérer la traversée de l'agglomération comme plus contraignante que celle de l'Atlantique en avion de ligne. Il peut se sentir plus proche d'un collègue new-yorkais que de l'habitant d'une banlieue à problèmes de la grande périphérie. La conscience des proximités potentielles est très influencée par la position sociale et l'activité.

La perception de la proximité relève donc à la fois des capacités financières des individus et de leurs représentations. Or celles-ci influencent aussi la 
manière dont une société produit son espace et le transforme (Gumuchian, 1991). Les politiques dont dépend l'aménagement de l'espace sont l'émanation du système de valeurs des décideurs et des représentations des élites. La priorité donnée aux liaisons rapides intermétropolitaines contribue à renforcer les inégalités d'accès aux proximités cumulées. La réduction de la distancetemps entre deux lieux $A$ et $B$ fera que $A$ et $B$ verront augmenter leur potentiel d'attractivité par effet induit, aux dépens des lieux intermédiaires. Cet « effet tunnel » est illustré par le système de l'étoile TGV que l'amélioration du réseau routier ne suffit pas à compenser.

L'inégalité des individus et des territoires face au progrès de la vitesse a fait de la proximité une notion éminemment relative. L'accessibilité potentielle n'est que très rarement utilisée par les ménages modestes, surtout dans les pays du «Sud». À São Paulo (17 millions d'habitants dont la moitié de pauvres), les riches évitent les énormes embouteillages, les enlèvements et les agressions en utilisant l'hélicoptère pour leurs déplacements d'affaires (héliports aux sommets des immeubles) ou pour rejoindre la famille dans la maison de vacances. Près de mille hélicoptères par jour sillonnent le ciel de la ville à $600 €$ l'heure...

La proximité doit être désormais pensée en fonction des flux et du mouvement. Les analyses convergent sur le constat que les sociétés contemporaines sont de moins en moins territoriales. Au commerce de proximité, celui du village ou du coin de la rue..., se substituent les hypermarchés et autres malls dont l'implantation dépend de l'intensité des flux : rocades, échangeurs, gares et stations de métro... Les consommateurs ne sont plus ceux du secteur d'implantation mais ceux qui passent et qui ne sont pas forcément toujours les mêmes. D'une certaine manière, quelques personnes sont proches de tout. La vie quotidienne s'organise alors selon d'autres formes de proximité, plus éclatées, à partir de lieux multiples constituant des «polygones de vie» différents les uns des autres. C'est un «espace en miettes». Nous vivons dans un monde de multi-appartenance spatiale. Les modes d'existence sont donc moins marqués par la proximité spatiale et ses symboles identitaires (églises, écoles, clubs sportifs...).

\section{Le voisinage}

Le proche, ce n'est donc plus la grande ville traditionnelle. On doit le construire ailleurs. C'est sur ce constat que se sont établis les deux principaux courants d'urbanisme contemporains, le fonctionnalisme et le mouvement des cités-jardins. Deux utopies, souvent présentées comme opposées mais qui sont en fait aussi antiurbaines l'une que l'autre. Dans les deux cas, une plus grande proximité est recherchée dans des unités d'habitat plus petites. 
La cité-jardin d'Ebenezer Howard (1902) est une solution qui combine les avantages de la campagne et ceux de la ville, sans en avoir les inconvénients : la proximité sans la jungle de la grande ville; la verdure sans l'isolement de la campagne. Il va marquer tout l'urbanisme contemporain et, notamment, l'idée de villes nouvelles. Le concept d' "unité de voisinage» de l'Américain Clarence Perry (1923) est très explicitement fondé sur le principe de proximité (tout le quotidien à 10 minutes de marche).

Le concept corbuséen d' «unité d'habitation » relève de la même logique de microville autonome mais développée verticalement, et repliée sur ses circulations intérieures. L'idée est de Fourier. Mais adaptée et popularisée par Le Corbusier, elle est à l'origine de nos grands ensembles, il est vrai sous des formes abâtardies. Le courant dissident du Team $X$ a produit des formes comme la galerie de l'Arlequin à Grenoble et le Mirail à Toulouse, qui ont fait rêver une génération d'urbanistes. C'est, dans les deux cas, d'une autre proximité qu'il s'agit, fondée sur l'idéalisation de la communauté équilibrée et de petite taille où les gens sont proches et solidaires, la Gemeinschaft de Tönnies. Cette communauté serait un remède aux maux de la grande ville symbole de la Gesellschaft (société de l'argent et de l'anonymat).

Mais quand l'utopie devient la réalité, le résultat est souvent décevant. Aux ghettos urbains que sont devenus certains grands ensembles répond la ségrégation délibérée des cités résidentielles privées (gated communities)... Ces fausses proximités spatiales donnent raison aux défenseurs de la rue traditionnelle, dont Jane Jacobs (1961), qui n'avaient pas attendu ces constats accablants pour prôner le voisinage plus spontané et imprévu de la ville dense, lieu de l'urbanité et des vraies proximités, contre les rénovations fonctionnalistes du West Side et l'idéal nauséeux des communautés de banlieue.

Les défis de l'aménagement urbain actuel consistent finalement à réintroduire dans ces «chantiers ratés » de la ville contemporaine un peu de la proximité des quartiers traditionnels. Encore une fois, les mots sont nouveaux, mais l'idée de proximité est toujours présente. Elle est exprimée par les politiques (plus que par les urbanistes) dans trois thèmes récurrents de ce que l'on a nommé, par abus de langage, la «politique de la ville», et avec des degrés variables selon les lieux et les périodes : 1 / la création de «centralité» pour introduire les avantages du centre dans des espaces de "relégation»; 2 / le «désenclavement » par le tramway ou le métro; 3 / la «mixité urbaine », enfin considérée comme un idéal incontournable, même si on a démontré depuis plus de trente ans que la proximité spatiale n'induit en rien une proximité relationnelle (Chamboredon et Lemaire, 1970). Il est vrai qu'il s'agit désormais de réintroduire la complexité urbaine dans des quartiers monofonctionnels. L'idée de complexité-proximité sociale était au demeurant déjà présente dans la notion de quartier («village dans la ville») dont la vogue n’a 
eu d'égal que le flou. Les valeurs de l'urbanité, de la mixité et de l'équilibre social sont finalement celles de la rue traditionnelle. On refait donc des rues, des ilots, de la ville dense ou compacte. Rien de bien nouveau...

L'ambigüité et la charge idéologique du mot "proximité» expliquent qu’il suscite la méfiance des spécialistes de l'espace. Mais ceci n'enlève rien à la pertinence du questionnement qu'il autorise. La concentration est une solution spontanée et illusoire à la question de la distance. Car la compétition qu'elle engendre exacerbe les inégalités et les «déséconomies», ce qui n'avait d'ailleurs pas échappé à A. Marshall. Dans l'habitat, la proximité spatiale ne fait plus illusion. Dans la ville contemporaine, les enjeux se sont d'ailleurs déplacés. La proximité comme synonyme de l'urbanité symbolisée par la ville dense existe encore mais n'est plus prépondérante. Elle est remplacée par une multitude de proximités choisies ou subies dans un espace urbain éclaté, champ de multiples espaces de vie. La mobilité devient la condition pour les maitriser. Il n'est d'ailleurs plus un colloque qui n'affiche ce thème. La mobilité fait de la proximité de tout le privilège d'une élite sociale (jet-set élargie) : proximité du centre-ville, proximité des autres villes mondiales, proximité des lieux de détente (du week-end en montagne au court séjour sous les «tropiques»)... Ce cumul des proximités potentielles est aussi l'apanage des métropoles. L'immobilité, la sédentarité sont synonymes d'éloignement et peuvent être de véritables stigmates sociaux (effet ghetto, ou «cité »; petites villes isolées des espaces non métropolisés), sauf quand elles sont revendiquées comme remèdes ou résistance à l'hypermobilité. À la revendication du «droit à la ville» (Lefebvre, 1968) se superpose donc désormais celle du «droit à la mobilité ». La proximité pose la question des rapports entre efficacité et justice ou performance économique et équité au sens de Rawls (1971). Le mot et son défi concernent les sciences de l'espace et de la ville. Mais ils sont d'abord une affaire de modèle de société. Ce qui est en cause c'est le statut du point de référence, celui du «centre», ville ou centre-ville, à partir desquels se déclinent tous les enjeux. Monocentrisme, polycentrisme ou absence de centre dominant (Allain, 2002). La question de la proximité est donc éminemment politique...

\section{Bibliographie}

ALONSO W. A., 1960, Location and Land Use. Toward a General Theory of Land Rent, Harvard University Press.

ALLAIN R., 2002, "Du concept au projet polycentrique», dans G. Baudelle et B. Castagnède (dir.), Le polycentrisme en Europe, La Tour d'Aigues, Éditions de l'Aube, Datar, chapitre 9.

CHAMBOREDON J. et LEMAIRE M., 1970, «Proximité spatiale et distance sociale. 
Les grands ensembles et leur peuplement », Revue française de sociologie, $\mathrm{n}^{\circ} 1$, p. 3-33.

CHRISTALLER W., 1933, Die zentralen Orte in Süddeutschland Central, traduit par C. W. Baskin, 1966, Places in Southern Germany, Englewood, Prentice Hall.

CLAVAL P., 1981, La logique des villes, Paris, Litec.

CONDILLAC É. (de), 1847 [1776], Le commerce et le gouvernement considérés relativement l'un à l'autre, réédité dans Mélanges d'économie politique, Paris, Daire et Molinari.

DELEUZE G. et GUATTARI F., 1980, Mille plateaux, Paris, Minuit.

ELIAS N., 1974, La société de cour, Paris, Calmann-Lévy.

GUMUCHIAN H., 1991, Représentations et aménagement du territoire, Paris, Anthropos, Economica.

HOWARD E., 1985 [réédition], Garden-Cities of To-Morrow, Eastbourne, Attic Books.

JACOBS J., 1961, The Death and Life of Great American Cities, Random House; traduit par C. Parin-Senemaud, 1991, Déclin et survie des grandes villes américaines, Sprimont (Belgique), Mardaga.

LEFEBVRE H., 1968, Le droit à la ville, Paris, Anthropos.

LEFEBVRE H., 1971, La révolution urbaine, Paris, Gallimard.

MARSHALL A., 1890, Principles of Economics, Londres, Macmillan and Co., traduit par F. Savaire-Jourdan, 1971, Principes d'économie, Paris, Librairie de droit et de jurisprudence.

MOLES A. et ROMER E., 1977, Psychologie de l'espace, Paris, Casterman.

OLLIVRO J., 2000, L'homme à toutes vitesses : de la lenteur homogène à la rapidité différenciée, Presses universitaires de Rennes.

RAWLS J., 1971, A Theory of Justice, Harvard University Press, traduit par C. Audard, 1987, Théorie de la justice, Paris, Le Seuil.

REILLY W. J., 1931, The Law of Retail Gravitation, New York, The Knickbroker Press.

REYNAUD A., 1981, Société, Espace, Justice, Paris, PUF.

ROGERS (Rapport), 1999, Towards an Urban Renaissance, Urban Task Force, Londres, E. \& F. N. Spon.

SMITH A., 1776, An Inquiry into the Nature and the Causes of the Wealth of Nations, traduit par Ph. Jaudel et J.-M. Servet, Recherche sur la nature et les causes de la richesse des nations, Paris, Economica, 2000.

VIRILIO P., 1996, Cybermonde, la politique du pire, Paris, Textuel.

WEBBER M., 1964, "The urban place and the nonplace urban realm », dans M. Webber et al., Exploration into Urban Structure, University of Pennsylvania Press, Philadelphie, traduit par X. Guillot et préfacé par F. Choay, 1996, L'urbain sans lieu ni bornes, La Tour d’Aigues, Éditions de l'Aube. 